\section{Effects of exercise on depression in old age}

The study by Mather et al (2002) is a laudable work but has some important shortcomings. The control group had received health education, and the authors have justified this approach. Considering the fact that well-designed studies addressing the usefulness of exercise in depression in old age are lacking, we believe inclusion of a control group that did not receive another intervention other than continuing antidepressants could have made Mather et al's findings more meaningful. Another issue is attendance rate: despite the fact that exercise facilitated recovery from depression and that no one dropped out, the reasons for a low mean attendance rate in the exercise group remain unclear. Also, whether this low attendance rate contributed to the lack of significant group differences in outcome measures at the 34th week (final assessment) needs clarification.

With regard to the statistical analysis, besides the analysis of outcome at certain points, the authors could have used the Wilcoxon test for paired samples or another comparable statistical test to detect differences in outcome from baseline scores. The authors state that both groups had scores of secondary outcome measures at the 10th and 34th weeks that were significantly different from baseline, but this statement is not supported by an appropriate statistical analysis.

Given that the authors had great difficulties while recruiting the study sample, rectification of the above limits could have made their conclusions more robust.

Mather, A. S., Rodriguez, C., Guthrie, M. F., et al (2002) Effects of exercise on depressive symptoms in older adults with poorly responsive depressive disorder. Randomised controlled trial. British Journal of Psychiatry I80, $4|I-4| 5$

K. Jagadheesan, S. Chakraborty, V. K. Sinha,

S. H. Nizamie Central Institute of Psychiatry,

Kanke (PO), Ranchi 834006, India

Authors' reply: We are grateful to Dr Jagadheesan and colleagues for allowing us to reiterate the methodological strength of our trial design. If our control group had been as Dr Jagadheesan proposes (continued antidepressant treatment only), then we would wrongly have concluded that exercise is highly effective as an adjunct to drug treatment in old age depression. In fact when compared with the effects of a structured social intervention, group exercise offered only a modest additional benefit. Our non-exercise control crucially allowed us to disentangle the psychosocial effects of coming together as a group from the effects of exercise itself. This novel use of a non-exercise control intervention which matched the exercise intervention in duration, frequency and social contact represents an important methodological advance which future researchers will wish to consider (Lawlor \& Hopker, 2001).

Perhaps Salmon may shed some light on the low mean attendance rate by his comment that advocacy of exercise as a treatment for depression 'must puzzle clinicians, who in treating depressed people, often have to contend with an absence of motivation to tackle much less strenuous features of life's routine' (Salmon, 1990).

The Results section of our paper is succinct, in part because of editorial constraints on article length. A typical finding (such as a group comparison of reduction in Hamilton Rating Scale for Depression score at 10 weeks) gives only a comparison of proportions and the associated $P$ value, with the statement 'we used Fisher's exact test in our comparison on the two groups' implied, but unwritten. In fact, we prided ourselves on the explicit statement of numerator and denominator here and elsewhere (23/42 $v$. 14/43) when many authors might simply have said ' $55 \% v$. $33 \%, P=0.05$.

We are surprised that Dr Jagadheesan et al advocate use of the Wilcoxon test for paired comparisons: this would have resulted in a highly undesirable plethora of comparisons (10 weeks $v$. baseline; 34 weeks $v$. baseline; 34 weeks $v$. 10 weeks). Statistical propriety necessitates the use of an approach which recognises the temporal ordering of the trio of results for each subject in each group; hence our appropriate use of repeated measures.

The analysis which resulted in the stated findings for secondary outcome measures was a one-sample $t$-test on the logarithms of the ratios of outcome:baseline scores.

There is a desperate need for betterquality research in the area of depression, and we believe that our trial design offers an important methodological advance.

\section{Declaration of interest}

M.E.T.M. is codirector of DD Developments, a University of Dundee company providing exercise classes for older people and whose profits support research into ageing.

Lawlor, D. A. \& Hopker, S.W. (200I) The effectiveness of exercise as an intervention in the management of depression: systematic review and meta-regression analysis of randomised controlled trials. BMY, 322. 763-767.

Salmon, P. (1990) Psychiatric benefits of physica exercise. British Journal of Hospital Medicine, 43, 107.

M. E. T. McMurdo, I. C. Reid Department of Medicine, University of Dundee, Ninewells Hospital and Medical School, Dundee DDI 9SY, UK

\section{Unethical use of placebo controls}

Klysner et al (2002) evaluated the prophylactic efficacy of citalopram in comparison with placebo in elderly patients, and stated 'the highly recurrent nature of major depression in the young and the elderly warrants long-term antidepressant treatment'. In view of this, is it ethical to use a placebo arm? The answer to this question depends upon whether or not there is an already available treatment of proven or accepted value. In this context, Cochrane (1989) stated that 'placebo controlled trials are appropriate when there is no existing treatment for a disorder, otherwise comparison trials are indicated. No new treatments should be introduced into medicine unless they have been shown, in randomised controlled trials, to be superior to existing treatments, or equivalent to existing treatment but cheaper or safer'. Similarly, section 12.4 of the National Health and Medical Research Council (1999) statement on ethical conduct in research involving humans states: 'the use of a placebo alone or the incorporation of a non-treatment control group is ethically unacceptable in a controlled trial where: (a) other available treatment has already been clearly shown to be effective; and (b) there is a risk of significant harm in the absence of treatment. If there is genuine uncertainty about the net clinical benefit of a treatment, a placebo controlled trial or a trial with a no-treatment arm may be considered'.

The use of placebo in this clinical drug trial raises questions of deception, of patient information and of informed consent. The patients in the placebo group were left without any active treatment for 48 weeks - this raises doubt as to whether patients were fully informed, before giving their consent, that they might receive a placebo by random allocation. We are keen to 
know why the authors did not try to compare the efficacy of citalopram with existing antidepressants.

Cochrane, A. L. (1989) Effectiveness and Efficiency: Random Reflections on Health Services. London: British Medical Journal.

Klysner, R., Bent-Hansen, J., Hansen H. L., et al (2002) Efficacy of citalopram in the prevention of recurrent depression in elderly patients: placebocontrolled study of maintenance therapy. British Journal of Psychiatry, 18I, 29-35.

National Health and Medical Research Council (1999) National Statement on Ethical Conduct in Research Involving Humans. Canberra: NHMRC.

A. K. Jainer St Michael's Hospital, St Michael's Road, South Warwickshire Primary Care Trust, South Warwickshire CV34 5QW, UK

N. Soni The Caludon Centre, Coventry Primary Care Trust, Coventry, UK

Authors'reply: Drs Jainer and Soni have addressed an important issue in clinical trials in depression when commenting on our article. Our study was the first specifically designed and conducted to evaluate the therapeutic value of prevention of recurrence of a depressive episode in an elderly population. The study was designed using the concept of the three phases of antidepressant treatment: acute, continuation and maintenance treatment (Montgomery et al, 1988). The study is unique in that the majority of the population had suffered only one documented depressive episode upon admission into the study.

At the time the study was initiated, there was sparse evidence for the value of prophylactic treatment after a first episode of depression in elderly patients. Thus, the requirement that there be no 'other available treatment [that] has already been clearly shown to be effective' was fulfilled.

Prior to initiating the study, the local ethics committee approved the protocol as well as the patient information and the informed consent form. The patient information explicitly mentioned the use of placebo in the double-blind period. All patients gave written informed consent before being included in the study.

Existing guidelines clearly stipulate that treatment of at least 6 months' duration is necessary to reduce the risk of relapse. The study complied with this by providing active treatment with citalopram for 24 weeks. Only patients in remission, after a total of 24 weeks of treatment with citalopram, were randomised to double-blind treatment with citalopram or placebo. The patients were closely monitored during the double-blind period until discontinuation or completion. Patients with recurrence of depression in the double-blind treatment period were withdrawn and treated at the investigators' discretion.

In addition, an active-comparator trial can only provide information regarding relative effect, but not whether prophylactic treatment is clinically warranted. The absolute value of prophylactic treatment can only be concluded from a placebocontrolled trial. Thus, the study had a placebo-controlled design for the doubleblind period, in accordance with the National Health and Medical Research Council guidelines as cited by Drs Jainer and Soni ('If there is a genuine uncertainty about the net clinical benefit of a treatment, a placebo controlled trial or a trial with a no-treatment arm may be considered').

The study established that long-term treatment with citalopram is effective in preventing recurrence of depression in the elderly and is well tolerated. With this knowledge, along with other currently available information, we certainly agree with the authors that the appropriateness of conducting similar studies in the future should be considered. However, our opinion notwithstanding, there is no consensus regarding the need for prophylactic treatment in the elderly. Until clinical practice and guidelines are changed, studies of a similar nature will have to be undertaken to convince the scientific community of the value of long-term treatment.

\section{Declaration of interest}

The study in question was funded by H. Lundbeck A/S. M.A. is an employee of H. Lundbeck A/S.

Montgomery, S. A., Dufour, H., Brion, S., et al (1988) The prophylactic efficacy of fluoxetine in unipolar depression. British Journal of Psychiatry, 153 (suppl. 3), 69-76.

R. Klysner Psychiatric Research Clinic, Frederiksberg Hospital, Denmark

M. Andersen International Clinical Research, H. Lundbeck A/S, Ottiliavej 9, 2500 Valby, Denmark

\section{Costs of dementia}

In their recent paper, Wolstenholme et al (2002) demonstrated that changes in cognitive and functional status have independent and significant effects on the costs of care in dementia. We agree with the authors that models of costs based solely on measures of cognitive changes are inappropriate to describe variables influencing the costs of dementia. From 1994 to 1999 we conducted in Italy a longitudinal study on costs of Alzheimer's disease (the CoDem Study), based on information obtained every 6 months from a sample of 148 patients with Alzheimer's disease living at home $(73.6 \%$ female, mean (s.d.) age 78 (7.8) years, mean (s.d.) Mini-Mental State Examination (MMSE) score at baseline 8.9 (8.3)), estimating direct and indirect costs of dementia (Trabucchi et al, 1996). In a preliminary analysis after the first year of observation, using a logistic regression analysis, we found that greater annual costs for Alzheimer's disease are significantly associated more with disability than with cognitive decline (Bianchetti et al, 1998). Following this line of investigation, we evaluated the modification of costs with the progression of the disease at the end of the 6-year longitudinal study with a Markov state transition model based on the comparison of costs for different states of cognitive and functional decline (measured using the MMSE and the Basic Activities of Daily Living (BADL) scale) (Jönsson et al, 1999). In our study total costs (per year) for dementia care varied from $€ 15450$ (£9972) for independent patients (BADL lost $=0$ ), to $€ 21463$ ( $£ 13853$ ) for partially independent subjects (1-3 BADL lost) and $€ 23762$ (£15336) for totally dependent patients (4-6 BADL lost). Using the MMSE, the costs varied from $€ 18024$ (£11633) for patients with mild Alzheimer's disease (MMSE > 20), to $€ 19665$ $(£ 12692)$ for patients with moderate decline (MMSE 15-20) and €25351 ( $£ 17077)$ for patients with severe cognitive decline (MMSE 8-14) (Trabucchi, 1999).

Our data, obtained in a sample of subjects with Alzheimer's disease living in a different social and cultural context, strengthen those obtained by Wolstenholme and colleagues, emphasising in particular the need to demonstrate an effect on functional status in the cost-effectiveness analysis of interventions in dementia.

\section{Declaration of interest}

The CoDem Study was funded by a grant from Bayer Pharmaceuticals, Milan, Italy. A.B. and M.T. have received financial support from various pharmaceutical 